\title{
LONGITUDINALLY INVARIANT ELASTIC STRUCTURES ANALYZED BY THE MESHLESS MLPG METHOD USING 2.5D APPROACH
}

\author{
P. Stanak ${ }^{1}$, A. Tadeu ${ }^{2}$, J. Sladek ${ }^{1}$, V. Sladek ${ }^{1}$
}

\begin{abstract}
This paper presents a general 2.5D meshless MLPG methodology for the computation of the elastic response of longitudinally invariant structure subjected to the incident wave field. A numerical frequency domain model is established using the Fourier transform in time and longitudinal coordinate domains. This allows for significant reduction of computational effort required. In the MLPG method the Moving-Least Squares (MLS) scheme is employed for the approximation of the spatial variation of displacement field. No finite elements are required for the approximation or integration of unknowns. A small circular subdomain is introduced around each nodal point in the analyzed domain. Local integral equations derived from the governing equations are specified on these subdomains. Continuously non-homogeneous material properties are varying in the cross-section of the analyzed structure. A simple patch test is introduced to assess the accuracy and the convergence of developed numerical model. At the end of the paper, numerical examples illustrate the applicability of the proposed numerical formulation.
\end{abstract}

Keywords: meshless method, 2.5D modeling, wave propagation, FGM materials

\section{Introduction}

The development of solution techniques for elastic wave propagation has been object of research over the years because the topic has a vast field of engineering applications in the field of civil and environmental engineering together with geophysical engineering or non-destructive analysis of materials and structures. Several reference works (Achenbach, 1973; Bedford and Drumheller, 1994) give a clear introduction to the topic of elastic wave propagation including nonhomogeneous solids (Berezovski et al., 2009).

The calculation of 3D elastic response of longitudinally invariant structures may become computationally demanding, unless more efficient techniques, such as 2.5D dimensional approach, are applied (Tadeu and Kausel, 2000). In case of 2.5D approach only the crosssection of the structure is discretized and Fourier series expansion is used to retrieve the results in the longitudinal direction. Longitudinally invariant structures are considered to be roads, railway tracks, tunnels, pipelines, dams or alluvial valleys (Francois et al., 2010). A wide range of tools is currently available for the analysis of elastic wave propagation in solid media including analytical techniques (Carcione et al., 1988) and numerical methods such as boundary element method (BEM) (Aliabadi, 2002; Tadeu, Kausel and Vrettos, 1996) or finite element method (FEM) (Zhang and Zhao, 1987). Tadeu and Antonio (2001) used 2.5D BEM approach

\footnotetext{
${ }^{1}$ Institute of Construction and Architecture, Slovak Academy of Sciences, Dúbravská cesta 9, 84503 Bratislava 45, Slovak Republic, e-mail: peter.stanak@savba.sk, jan.sladek@savba.sk,vladimir.sladek@savba.sk

2 ITeCons, University of Coimbra, Pólo II, Rua Pedro Hispano, 3030-289, Coimbra, Portugal
} 
for the layered elastic and acoustic domains. 2.5D FEM was applied by Hung et al. (2013) for the analysis of soil vibrations along the railway due to moving trains. Sheng et al. (2006) proposed a wave-number finite/boundary element method for predicting the vibration spectra for circular tunnel structures. Even though the FEM and BEM have encountered wide acceptance and success on a commercial market, they possess some drawbacks such as unavailability of fundamental solution for certain problems at BEM or locking of elements, stress discontinuity across elements or costly remeshing in large problems with moving boundaries related to FEM.

In recent time, a class of numerical approaches known as meshless methods has attracted considerable attention due to its potential to solve the above-mentioned. Among many meshless or meshfree methods available, the meshless local Petrov-Galerkin (MLPG) method (Atluri et al., 2000; Atluri, 2004) has received considerable scientific attention. MLPG is a truly meshless method, thus no elements are required neither for approximation nor for integration of unknowns. Meshless methods are also advantageous by their ability to efficiently treat problems with continuously non-homogeneous domains, since the unknown field quantities are approximated only in terms of nodes instead of elements used in mesh-based methods such as the FEM, thus the continuous variation of material properties is maintained exactly. Sladek, Sladek and Zhang (2003) applied the MLPG for the elastodynamic problems in continuously non-homogeneous bodies. Acoustic wave propagation in non-homogeneous media was analyzed by coupled BEM-MLPG approach in (Tadeu et al., 2014). The MLPG method was applied for analysis of axisymmetric continuously non-homogeneous piezoelectric solids (Sladek et al., 2008), fracture analysis (Sladek et al. 2009) or circular plates under transient loading (Stanak et al. (2011); Sladek et al., 2013a). The review articles by Dong et al. (2014) and Sladek et al. (2013b) describe the principles of the MLPG method and its application to analyses of a broad range of scientific problems.

In the present contribution, the MLPG formulation is used for the modeling of the displacement responses of the 2.5D nonhomogeneous elastic domain. The analysis is performed in the frequency domain with the use of variables in the form of complex numbers. Nodal points are spread on the analyzed 2D domain without any restrictions. A small local circular subdomain is introduced around each nodal point. Local integral equations (LIEs) constructed from governing PDEs are defined over these circular subdomains. Moving Least-Squares (MLS) approximation scheme (Lancaster and Salkauskas, 1981) is used to approximate the spatial variations of electric and mechanical fields. The essential boundary conditions are satisfied by the collocation of MLS approximation expressions for prescribed displacements on boundary nodes.

Hollow cylinder with nonhomogeneous material properties is analyzed as a numerical example to demonstrate the applicability of present method. The patch test is first introduced to evaluate the accuracy of proposed formulation. Then non-uniform loading in the vertical direction is considered. The results at the cross-section of the cylinder are obtained from the nodal points while the results along longitudinal z-coordinate are computed using the Fourier series. Finally, some conclusions are given and the quality of the numerical results is discussed.

\section{Governing equations for elastic wave propagation}

Specification of the considered problem requires a mathematical formulation of governing equations for elastic wave propagation in nonhomogeneous solids. For the general 3D problem with $i, j=1,2,3$ one can define the following equilibrium equation 


$$
\sigma_{i j, j}(\mathbf{x}, t)=\rho \ddot{u}_{i}(\mathbf{x}, t)
$$

where $\sigma_{i j}, u_{i}, \rho$ is the stress tensor, the mechanical displacements and the mass density, respectively. A comma followed by an index denotes partial differentiation with respect to the spatial coordinate $x_{i}$. The dots over the quantity indicate the derivative with respect to time $t$.

Let us consider a cylindrical body with boundary conditions on the lateral sides as well as the material properties of the structure being invariant along the longitudinal $z$-direction $\left(x_{3} \equiv z\right)$. Then it is appropriate to perform the 2.5D analysis. Considering the Fourier transform in both frequency and spatial domain gives

$$
\tilde{f}\left(\omega, k_{z}\right)=\int_{-\infty}^{\infty} \int_{-\infty}^{\infty} f(t, z) e^{-\mathrm{i}\left(k_{z} z-\omega t\right)} d t d z
$$

where $\omega$ is the angular frequency, $k_{z}$ is the axial wave number and a dependence of the type $e^{-\mathrm{i}\left(k_{z} z-\omega t\right)}$ is implicit.

Applying the Fourier transform (2) to Eq. (1) and separating the third spatial component (thus $\alpha=1,2$ ), one may obtain

$$
\tilde{\sigma}_{i \alpha, \alpha}\left(\mathbf{x}, k_{z}, \omega\right)+\mathrm{i} k_{z} \tilde{\sigma}_{i 3}\left(\mathbf{x}, k_{z}, \omega\right)=-\omega^{2} \rho \tilde{u}_{i}\left(\mathbf{x}, k_{z}, \omega\right)
$$

As $\mathbf{x} \equiv\left(x_{1}, x_{2}\right)$, all physical quantities may vary at the cross-sectional plane.

The elastic stress tensor is defined as

$$
\tilde{\sigma}_{i j}=\lambda \delta_{i j} \tilde{u}_{k, k}+\mu\left(\tilde{u}_{i, j}+\tilde{u}_{j, i}\right)
$$

where $\lambda, \mu$ are the Lame's material constants and $\delta_{i j}$ is the Kronecker delta symbol. In certain problems the non-homogeneity of isotropic material is observed with varying Young's modulus $E(\mathbf{x})$ while the Poisson's ratio $v$ is considered to be constant. Then the varying Lame's constants can be defined as

$$
\mu(\mathbf{x})=\frac{E(\mathbf{x})}{2(1+v)}, \lambda(\mathbf{x})=\frac{E(\mathbf{x}) v}{(1+v)(1-2 v)}
$$

\section{Local integral equations for the MLPG method}

For the meshless analysis of elastic wave propagation in the analyzed domain, the MLPG is chosen, assuming the MLS approximation for the definition of the trial functions and Heaviside unit step function as a test function in each local subdomain $\Omega_{S}$, see (Atluri, 2004). Instead of writing the global weak form, the MLPG is based on the local weak form of the governing equations. The local integration domain $\Omega_{S}$ can have arbitrary shape, thus the cylindrical shape aligned in the longitudinal $z \equiv 3$ direction is considered in this case. Since the problem is assumed to be infinite in the longitudinal direction, the volume integral can be decomposed as 
integral over the z-coordinate and the cross section of cylinder $\Omega_{S}$. The local integration domain $\Omega_{S}$ is shown in Fig. 1 for $2 \mathrm{D}$ case.

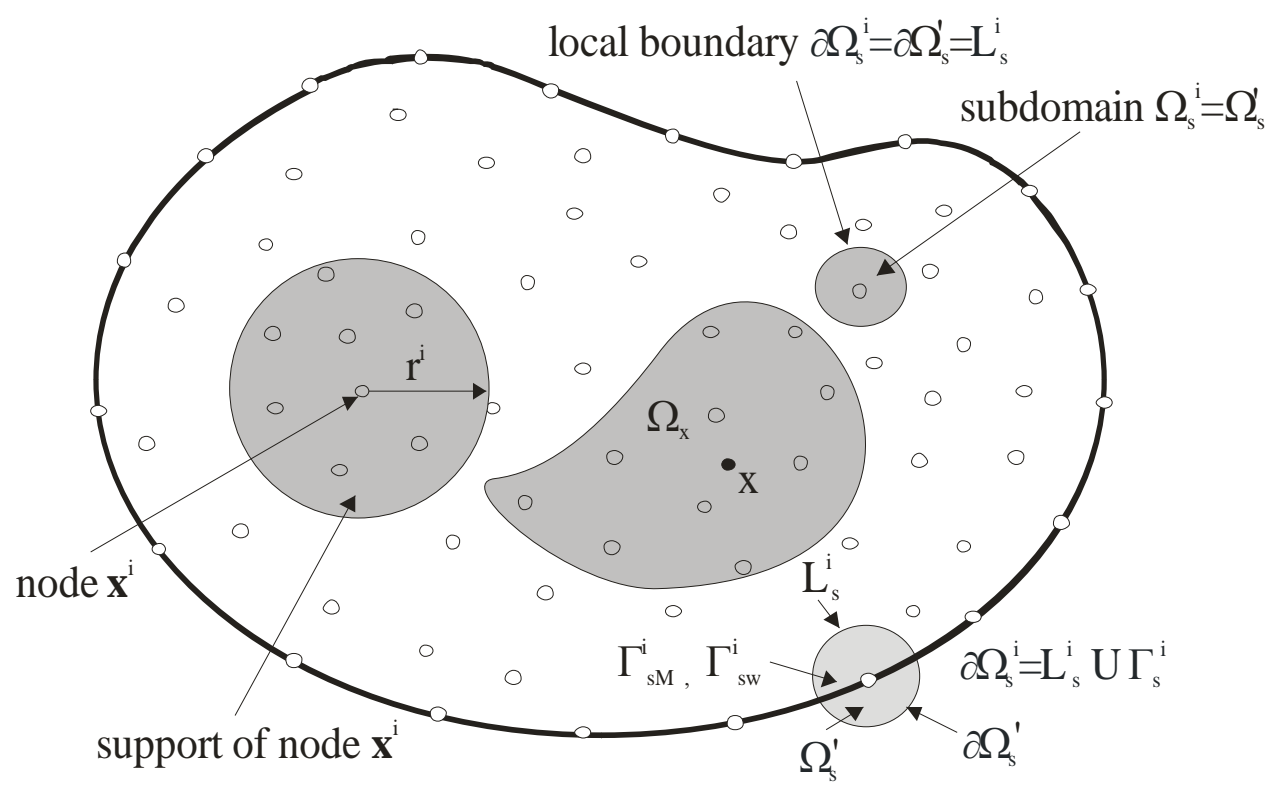

Figure 1. Local boundaries for weak formulation, the domain $\Omega_{x}$ for MLS approximation of the trial function, and support area of weight function around node

The local weak form of Eq. (3) is then written over each subdomain $\Omega_{S}$ as

$$
\int_{\Omega_{S}}\left[\tilde{\sigma}_{i \alpha, \alpha}\left(\mathbf{x}, k_{z}, \omega\right)+\mathrm{i} k_{z} \tilde{\sigma}_{i 3}\left(\mathbf{x}, k_{z}, \omega\right)+\omega^{2} \rho \tilde{u}_{i}\left(\mathbf{x}, k_{z}, \omega\right)\right] w^{*}(\mathbf{x}) d \Omega=0
$$

It is convenient to apply the Gauss divergence theorem for the left hand side integral in Eq. (6) that leads to

$$
\begin{aligned}
& \int_{\partial \Omega_{S}} n_{\alpha}(\mathbf{x}) \tilde{\sigma}_{i \alpha}\left(\mathbf{x}, k_{z}, \omega\right) w^{*}(\mathbf{x}) d \Gamma-\int_{\Omega_{S}} \tilde{\sigma}_{i \alpha}\left(\mathbf{x}, k_{z}, \omega\right) w_{, \alpha}^{*}(\mathbf{x}) d \Omega \\
& +\int_{\Omega_{S}}\left[\mathrm{i} k_{z} \tilde{\sigma}_{i 3}\left(\mathbf{x}, k_{z}, \omega\right)+\omega^{2} \rho \tilde{u}_{i}\left(\mathbf{x}, k_{z}, \omega\right)\right] w^{*}(\mathbf{x}) d \Omega=0
\end{aligned}
$$

where $n_{j}(\mathbf{x})$ is the unit normal vector and $\partial \Omega_{S}$ is the boundary of the subdomain $\Omega_{S}$. Assuming the Heaviside unit step function for the test function

$$
w^{*}(\mathbf{x})=\left\{\begin{array}{lll}
1 & \text { at } & \mathbf{x} \in\left(\Omega_{s} \cup \partial \Omega_{s}\right) \\
0 & \text { at } & \mathbf{x} \notin\left(\Omega_{s} \cup \partial \Omega_{s}\right)
\end{array}\right.
$$


the following local integral equation (LIE) is finally obtained

$$
\int_{\partial \Omega_{S}} n_{\alpha}(\mathbf{x}) \tilde{\sigma}_{i \alpha}\left(\mathbf{x}, k_{z}, \omega\right) d \Gamma+\int_{\Omega_{S}}\left[\mathrm{i} k_{z} \tilde{\sigma}_{i 3}\left(\mathbf{x}, k_{z}, \omega\right)+\omega^{2} \rho \tilde{u}_{i}\left(\mathbf{x}, k_{z}, \omega\right)\right] d \Omega=0 .
$$

Integrand in the first boundary integral in Eq. (9) can be identified as the Fourier transform of the traction vector $\tilde{t}_{i}\left(\mathbf{x}, k_{z}, \omega\right)=n_{\alpha}(\mathbf{x}) \tilde{\sigma}_{i \alpha}\left(\mathbf{x}, k_{z}, \omega\right)$.

In general, a meshless method uses a local interpolation to represent the trial function with the values (or the fictitious values) of the unknown variable at some randomly located nodes. For the approximation of trial functions various approximation schemes such as radial basis functions (RBF), partition of unity (PU), reproducing kernel particle methods (RKPM) or moving least-squares (MLS) can be used within the framework of MLPG method (Atluri, 2004). The moving least-squares (MLS) approximation used in our analysis may be considered as one of such schemes. According to the MLS method (Atluri, 2004), the approximation of displacements $\tilde{u}_{i}\left(\mathbf{x}, k_{z}, \omega\right)=\tilde{\mathbf{u}}\left(\mathbf{x}, k_{z}, \omega\right)$ by approximant $\tilde{\mathbf{u}}^{h}\left(\mathbf{x}, k_{z}, \omega\right)$ is given by

$$
\tilde{\mathbf{u}}\left(\mathbf{x}, k_{z}, \omega\right) \cong \tilde{\mathbf{u}}^{h}\left(\mathbf{x}, k_{z}, \omega\right)=\sum_{a=1}^{N} \phi^{a}(\mathbf{x}) \hat{\mathbf{u}}^{a}\left(k_{z}, \omega\right)
$$

where the nodal values $\hat{\mathbf{u}}^{a}\left(k_{z}, \omega\right)$ are called fictitious nodal parameters of the mechanical displacements. The MLS shape function $\phi^{a}(\mathbf{x})$ is defined over a set of $N$ nodes located in the support domain $\Omega_{x}$ (Atluri, 2004) as shown in Fig.1. $C^{1}$-continuity of the MLS approximation is ensured by the fourth-order spline type weight function used for the construction of the shape function given as

$$
v^{a}(\mathbf{x})=\left\{\begin{array}{cc}
1-6\left(\frac{d^{a}}{r^{a}}\right)^{2}+8\left(\frac{d^{a}}{r^{a}}\right)^{3}-3\left(\frac{d^{a}}{r^{a}}\right)^{4} & 0 \leq d^{a} \leq r^{a} \\
0 & d^{a} \geq r^{a}
\end{array}\right.
$$

where $d^{a}=\left\|\mathbf{x}-\mathbf{x}^{a}\right\|$ and $r^{a}$ is the radius of the circular support domain. The value of $N$ in Eq. (10) is determined by the number of nodes lying in the support domain with radius $r^{a}$. The partial derivatives of field quantities are approximated with the use of the shape function derivative $\phi_{, \alpha}^{a}(\mathbf{x})$ and same fictitious parameters as

$$
\tilde{\mathbf{u}}_{, \alpha}\left(\mathbf{x}, k_{z}, \omega\right)=\sum_{a=1}^{N} \phi_{, \alpha}^{a}(\mathbf{x}) \hat{\mathbf{u}}^{a}\left(k_{z}, \omega\right)
$$

Note that derivative in the longitudinal direction is defined as

$$
\tilde{\mathbf{u}}_{, 3}\left(\mathbf{x}, k_{z}, \omega\right)=\mathrm{i} k_{z} \sum_{a=1}^{N} \phi^{a}(\mathbf{x}) \hat{\mathbf{u}}^{a}\left(k_{z}, \omega\right)
$$


Applying Eqs. $(10,12,13)$ to approximation of trial functions $\tilde{\mathbf{u}}\left(\mathbf{x}, k_{z}, \omega\right)$ and their derivatives to Fourier transformed stress tensor (4) and its subsequent insertion into local integral equation (9) is leading to a set of discretized local integral equations in the following component form

$$
\begin{aligned}
& \sum_{a=1}^{N} \hat{u}_{1}^{a}\left(k_{z}, \omega\right) \int_{\partial \Omega_{S}}\left[(\lambda+2 \mu) n_{1}(\mathbf{x}) \phi_{, 1}^{a}(\mathbf{x})+n_{2}(\mathbf{x}) \mu \phi_{, 2}{ }^{a}(\mathbf{x})\right] d \Gamma+ \\
& +\sum_{a=1}^{N} \hat{u}_{2}^{a}\left(k_{z}, \omega\right) \int_{\partial \Omega_{S}}\left[n_{1}(\mathbf{x}) \lambda \phi_{, 2}^{a}(\mathbf{x})+n_{2}(\mathbf{x}) \mu \phi_{, 1}^{a}(\mathbf{x})\right] d \Gamma+ \\
& +i k_{z} \lambda \sum_{a=1}^{N} \hat{u}_{3}^{a}\left(k_{z}, \omega\right) \int_{\partial \Omega_{S}} n_{1}(\mathbf{x}) \phi^{a}(\mathbf{x}) d \Gamma+\left[\mu\left(i k_{z}\right)^{2}+\omega^{2} \rho\right] \sum_{a=1}^{N} \hat{u}_{1}^{a}\left(k_{z}, \omega\right) \int_{\Omega_{S}} \phi^{a}(\mathbf{x}) d \Omega+ \\
& +i k_{z} \mu \sum_{a=1}^{N} \hat{u}_{3}^{a}\left(k_{z}, \omega\right) \int_{\Omega_{S}} \phi_{, 1}^{a}(\mathbf{x}) d \Omega=0 \\
& \sum_{a=1}^{N} \hat{u}_{1}^{a}\left(k_{z}, \omega\right) \int_{\partial \Omega_{S}}\left[n_{2}(\mathbf{x}) \lambda \phi_{, 1}^{a}(\mathbf{x})+n_{1}(\mathbf{x}) \mu \phi_{, 2}^{a}(\mathbf{x})\right] d \Gamma+ \\
& +\sum_{a=1}^{N} \hat{u}_{2}^{a}\left(k_{z}, \omega\right) \int_{\partial \Omega_{S}}\left[(\lambda+2 \mu) n_{2}(\mathbf{x}) \phi_{, 2}^{a}(\mathbf{x})+n_{1}(\mathbf{x}) \mu \phi_{, 1}^{a}(\mathbf{x})\right] d \Gamma+ \\
& +i k_{z} \lambda \sum_{a=1}^{N} \hat{u}_{3}^{a}\left(k_{z}, \omega\right) \int_{\partial \Omega_{S}} n_{2}(\mathbf{x}) \phi^{a}(\mathbf{x}) d \Gamma+\left[\mu\left(i k_{z}\right)^{2}+\omega^{2} \rho\right] \sum_{a=1}^{N} \hat{u}_{2}^{a}\left(k_{z}, \omega\right) \int_{\Omega_{S}} \phi^{a}(\mathbf{x}) d \Omega+ \\
& +i k_{z} \mu \sum_{a=1}^{N} \hat{u}_{3}^{a}\left(k_{z}, \omega\right) \int_{\Omega_{S}} \phi_{, 2}^{a}(\mathbf{x}) d \Omega=0 \\
& i k_{z} \mu \sum_{a=1}^{N} \hat{u}_{1}^{a}\left(k_{z}, \omega\right) \int_{\partial \Omega_{S}} n_{1}(\mathbf{x}) \phi^{a}(\mathbf{x}) d \Gamma+i k_{z} \mu \sum_{a=1}^{N} \hat{u}_{2}^{a}\left(k_{z}, \omega\right) \int_{\partial \Omega_{S}} n_{2}(\mathbf{x}) \phi^{a}(\mathbf{x}) d \Gamma+ \\
& +\mu \sum_{a=1}^{N} \hat{u}_{3}^{a}\left(k_{z}, \omega\right) \int_{\partial \Omega_{S}}\left[n_{1}(\mathbf{x}) \phi_{, 1}^{a}(\mathbf{x})+n_{2}(\mathbf{x}) \phi_{, 2}^{a}(\mathbf{x})\right] d \Gamma+ \\
& +i k_{z} \lambda \sum_{a=1}^{N} \hat{u}_{1}^{a}\left(k_{z}, \omega\right) \int_{\Omega_{S}} \phi_{, 1}^{a}(\mathbf{x}) d \Omega+i k_{z} \lambda \sum_{a=1}^{N} \hat{u}_{2}^{a}\left(k_{z}, \omega\right) \int_{\Omega_{S}} \phi_{, 2}^{a}(\mathbf{x}) d \Omega+ \\
& +\left[\omega^{2} \rho+\left(i k_{z}\right)^{2}(\lambda+2 \mu)\right] \sum_{a=1}^{N} \hat{u}_{3}^{a}\left(k_{z}, \omega\right) \int_{\Omega_{S}} \phi^{a}(\mathbf{x}) d \Omega=0
\end{aligned}
$$


A collocation approach is used to impose essential boundary conditions directly, using MLS variable approximation (10). For natural boundary conditions local integral equations are written for the nodes on the appropriate segments of the global boundary as explained in (Atluri, 2004).

\section{The patch test - verification and accuracy of the proposed model}

Development of new computational methods for solving complex problems such as elastic wave propagation requires tools for checking the quality of computed results. The patch test can be considered as one of such numerical procedures to address the convergence of the applied method. Convergence, in sense of numerical computing, describes how the results, under specific conditions, approach the exact solution. The patch test was developed primarily for the finite element method (FEM) however it can also be used for other computational methods including MLPG as shown in (Stanak et al., 2012).

In the present case, the known displacement field is prescribed on the boundary nodes using collocation of MLS approximation expression (10). A homogeneous cylinder having radius $1.0 \mathrm{~m}$ is considered for the analysis. Only the circular cross-section centered at $\left(x_{1 c e n}=0.0 \mathrm{~m} ; x_{2 c e n}=0.0 \mathrm{~m}\right)$ is discretized using 701 internal nodal points and 100 nodes on the boundary. The cylinder is excited by a line load whose amplitude varies sinusoidally in the third dimension $\left(k_{z}=0.3 \mathrm{rad} / \mathrm{m}\right)$ located at a given point $\left(x_{1}^{0}=-4.0 \mathrm{~m} ; x_{2}^{0}=0.0 \mathrm{~m}\right)$, in the outer homogeneous domain with the same material parameters. The medium, with an elasticity modulus of $E=11689288.6 \mathrm{kPa}$, Poisson ratio of $v=0.29593$ density of $\rho=2140 \mathrm{~kg} / \mathrm{m}^{3}$ is considered. The incident displacement field applied on the boundary is given as

$$
\begin{aligned}
& u_{1}^{i n c}\left(\mathbf{x}^{0}, \mathbf{x}_{s}, k_{z}, \omega\right)=\frac{\mathrm{i}}{2} k_{c_{p}} H_{1}\left(k_{c_{p}} r\right) \frac{\partial r}{\partial x_{1}} \\
& u_{2}^{i n c}\left(\mathbf{x}^{0}, \mathbf{x}_{s}, k_{z}, \omega\right)=\frac{\mathrm{i}}{2} k_{c_{p}} H_{1}\left(k_{c_{p}} r\right) \frac{\partial r}{\partial x_{2}} \\
& u_{3}^{\text {inc }}\left(\mathbf{x}^{0}, \mathbf{x}_{s}, k_{z}, \omega\right)=\frac{k_{z}}{2} H_{0}\left(k_{c_{p}} r\right)
\end{aligned}
$$

with $\quad r=\sqrt{\left(\mathrm{x}_{1}^{0}-\mathrm{x}_{1 s}\right)^{2}+\left(\mathrm{x}_{2}^{0}-\mathrm{x}_{2 s}\right)^{2}}$ and the dilatational wave number $k_{c_{p}}=\sqrt{\omega^{2} / c_{p}{ }^{2}-k_{z}^{2}}$, assuming $\operatorname{Im}\left(k_{c_{p}}\right)<0 . H_{n}(\ldots)$ are second kind Hankel functions of the order $n$ and $c_{p}$ is the dilatational wave velocity.

Figure 2 presents the uniform distribution of the 100 boundary nodes and 701 internal nodes used to compute the solution. The radius of the MLS support domain is chosen as three times distance between two neighboring nodes. Results for a harmonic source with the frequency $100 \mathrm{~Hz}$ are presented next to illustrate the accuracy of the solution. 
Using the exact benchmark solution, one can compute relative errors of the numerical analysis. Relative errors are computed for displacements $u_{1}$. The relative error for every node is specified in percentage as

$$
e=100 \frac{\left|u_{1}^{\text {num }}-u_{1}^{\text {exact }}\right|}{\left|u_{1}^{\text {exact }}\right|}[\%]
$$

where $u_{1}^{\text {num }}$ represents the computed value of axial displacement and $u_{1}^{\text {exact }}$ represents "exact" solution as obtained by straightforward use of Eq. (17) for each nodal point. Figure 2 shows the nodal distribution of total 801 nodes used for the calculations. Figure 3 then presents the resulting distribution of real and imaginary part of displacement $u_{1}^{\text {num }}$.

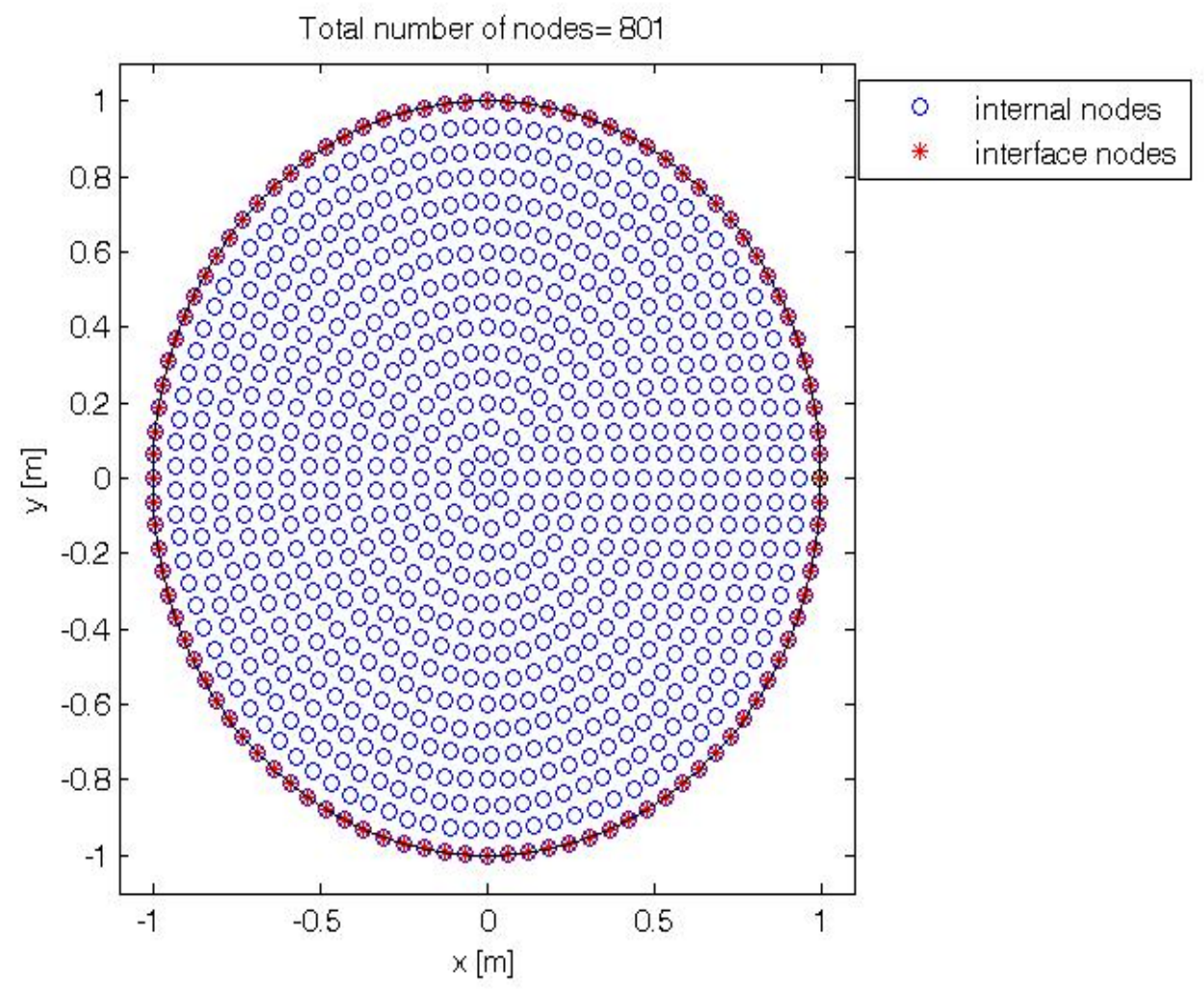

Figure 2. Nodal distribution for the considered problem 

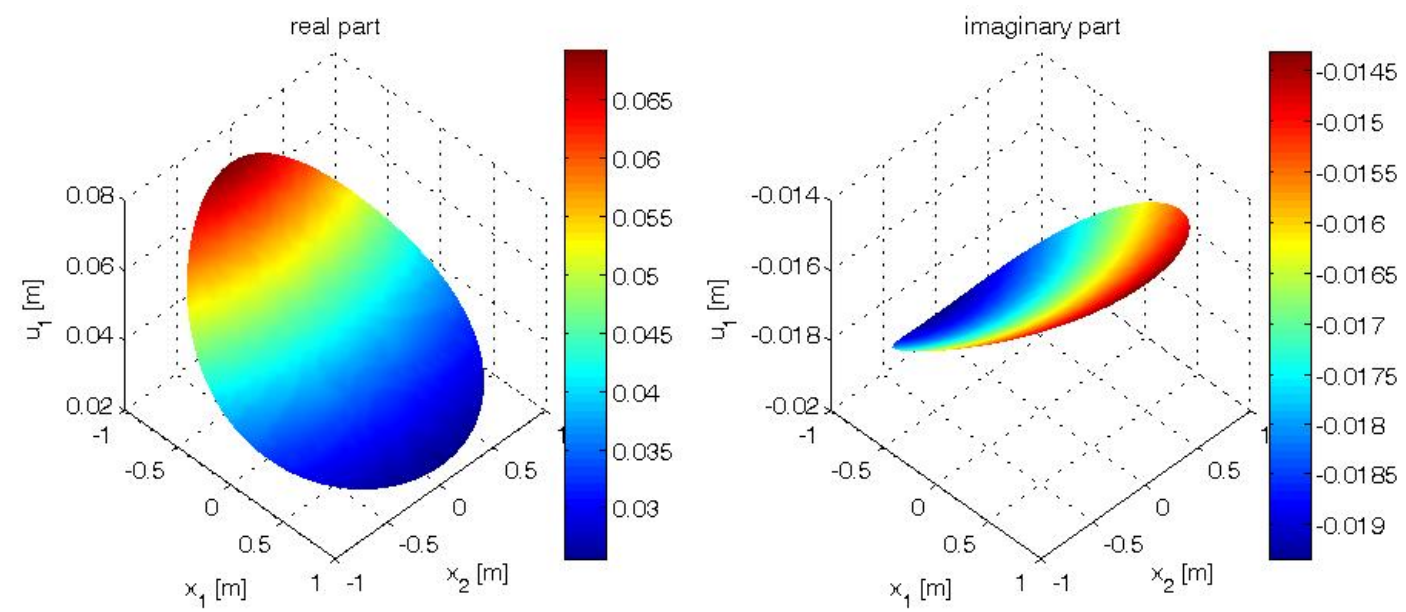

Figure 3. Resulting real (left) and imaginary (right) parts of displacement $u_{1}^{\text {num }}$ obtained for incident load with frequency $100 \mathrm{~Hz}$
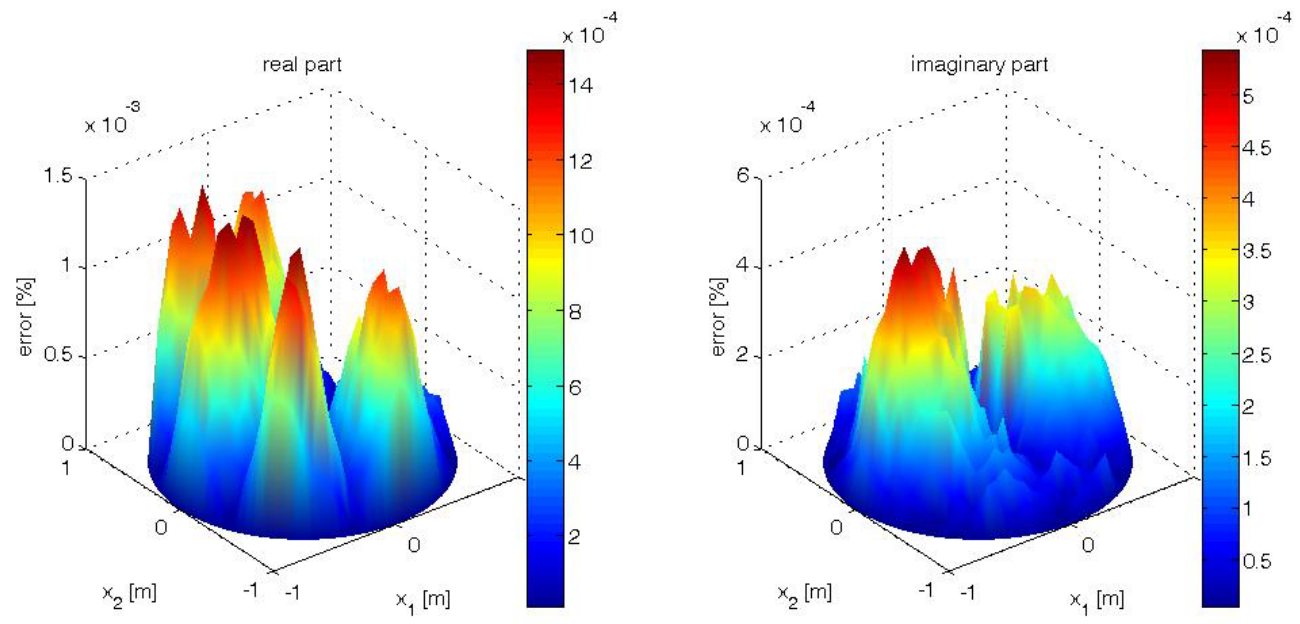

Figure 4. Relative error distribution for real (left) and imaginary (right) part of computed displacement $u_{1}^{\text {num }}$

Finally, the relative error distribution for the displacement $u_{1}^{\text {num }}$ is given in Figure 4 . One may observe that the error tends to zero close to the boundary since on the boundary nodes the exact values $u_{1}^{\text {exact }}$ are prescribed directly. Note that the errors are quite small at the order of $1 \times 10^{-3} \%$. 
It may be convenient to specify other type of error estimation that would tackle the error of the numerical solution from the global viewpoint. Averaged percentage error (Sladek et al., 2005) was introduced for this reason as

$$
A E=100 \frac{\left\|u_{i}^{\text {num }}-u_{i}^{\text {exact }}\right\|}{\left\|u_{i}^{\text {exact }}\right\|}[\%]
$$

where the norm is given as $\|*\|=\left(\sum_{a=1}^{N_{t}}\left[*\left(\mathbf{x}^{a}\right)\right]^{2}\right)^{\frac{1}{2}}$ and $N_{t}$ is the total number of nodes used in the analysis. Using this error estimate the convergence of the method for varying nodal densities can be determined as shown in Fig. 5. Results show that the longitudinal displacement $u_{3}$ is reaching the best accuracy than the remaining two; however the convergence rate for both quantities is almost the same. The errors for the displacement $u_{2}$ can be considered the highest because the source point of the load $\mathbf{x}^{0}$ lies on the $x$-axis. Thus the resulting displacements $u_{2}$ are almost zero over the nodes lying on the $x$-axis which lead to increased errors. This numerical test showed that the developed formulation passed the patch test with positive convergence for increased number of nodes.

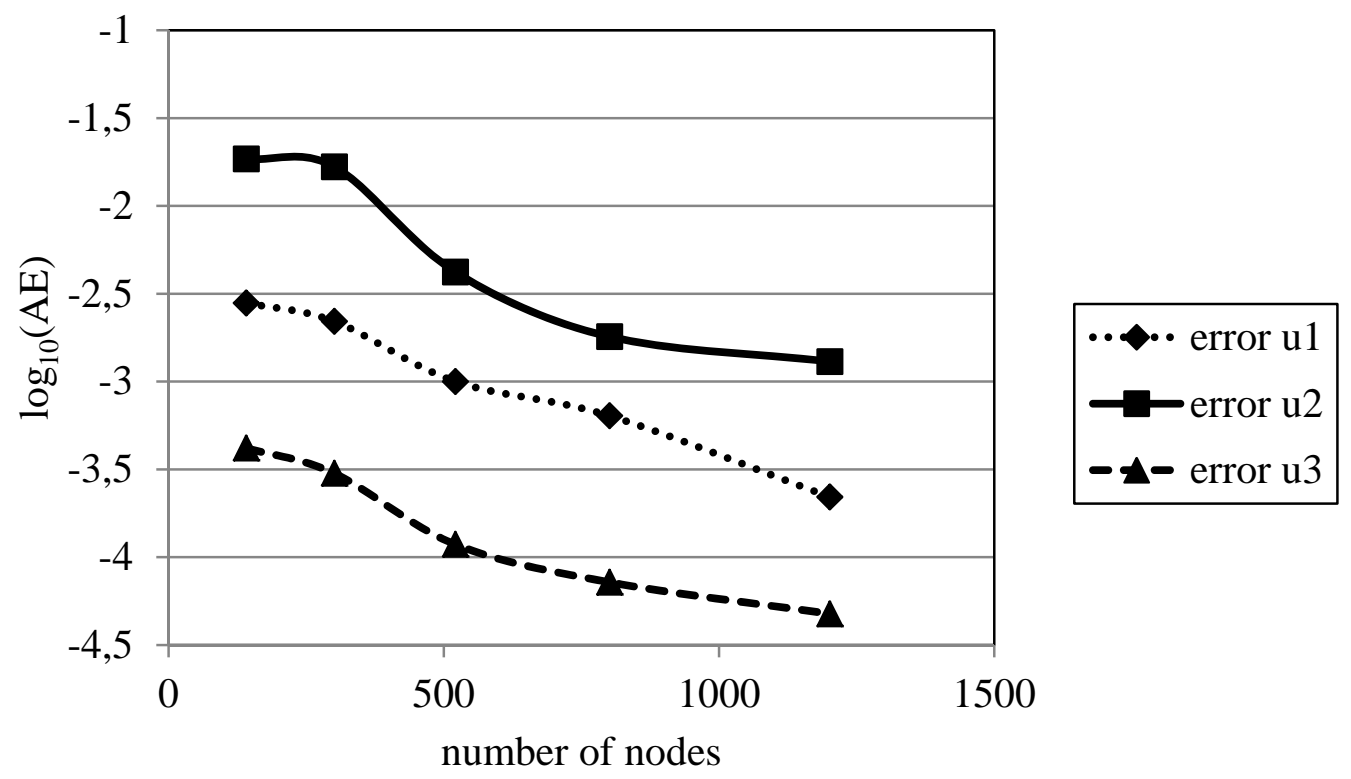

Figure 5. Averaged relative error distribution and the convergence rate 


\section{Numerical examples}

Results of numerical experiments are presented in this section to illustrate the applicability of the proposed MLPG formulation for a more general problem. The analysis of a hollow cylinder (pipe) with continuously nonhomogeneous material properties subjected to non-uniform load is considered. Schematic visualization of the geometry is shown in Fig. 6. The hollow interior with diameter $R_{\text {in }}=0.33 \mathrm{~m}$ is considered rigid (with zero prescribed displacements). On the outer boundary with the diameter $R_{\text {out }}=1.0 \mathrm{~m}$ is prescribed the non-uniform traction

$$
\tilde{t}_{2}^{i}(\mathbf{x})=\operatorname{sign}\left(x_{2}^{i}(\mathbf{x})\right) \tilde{t}_{0} \cos \left(\frac{\pi}{2} \frac{x_{1}^{i}(\mathbf{x})}{R_{\text {out }}}\right)
$$

where $\tilde{t}_{0}=1000 \mathrm{~N} / \mathrm{m}^{2}$ is the maximum applied load. This applied load varies sinusoidally in the longitudinal direction with $k_{z}=0.3 \mathrm{rad} / \mathrm{m}$ and frequency $1000.0 \mathrm{~Hz}$.

Nonhomogeneous material properties of the cylinder are defined through continuous variation of Young's modulus $E^{i}(\mathbf{x})$ as

$$
E^{i}(\mathbf{x})=E_{0}+E_{0} \frac{2 x_{2}^{i}(\mathbf{x})}{r} e^{\left(-\left(\frac{2 x_{1}^{i}(\mathbf{x})}{r}\right)^{2}-\left(\frac{2 x_{2}^{i}(\mathbf{x})}{r}\right)^{2}\right)}
$$

where $E_{0}$ is the initial value of Young's modulus, $x_{1}^{i}(\mathbf{x}), x_{2}^{i}(\mathbf{x})$ are the nodal coordinates and $r$ is the radius of the cylinder. Variation of Lame's constants that depend on $E^{i}(\mathbf{x})$ is shown in Fig. 7.

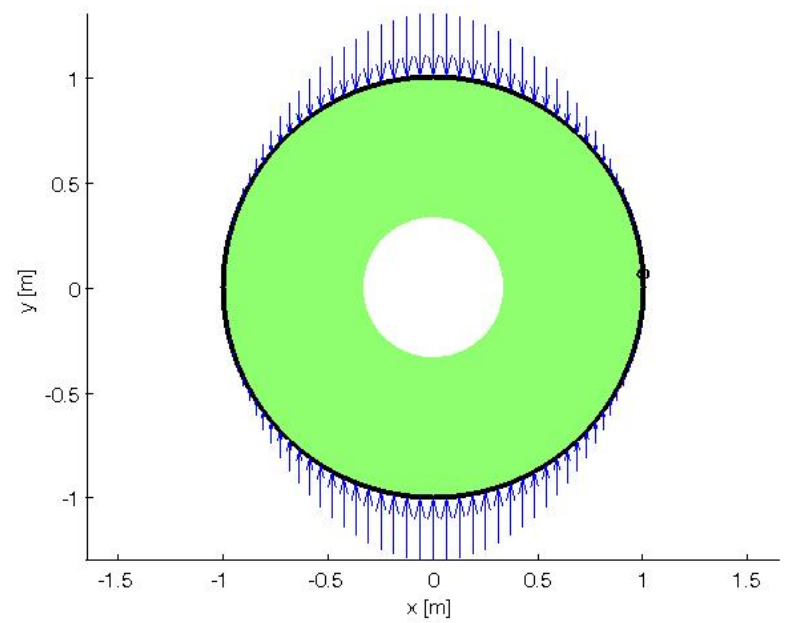

Figure 6. Schematic representation of hollow cylinder with the pattern of the traction load indicated by blue arrows 


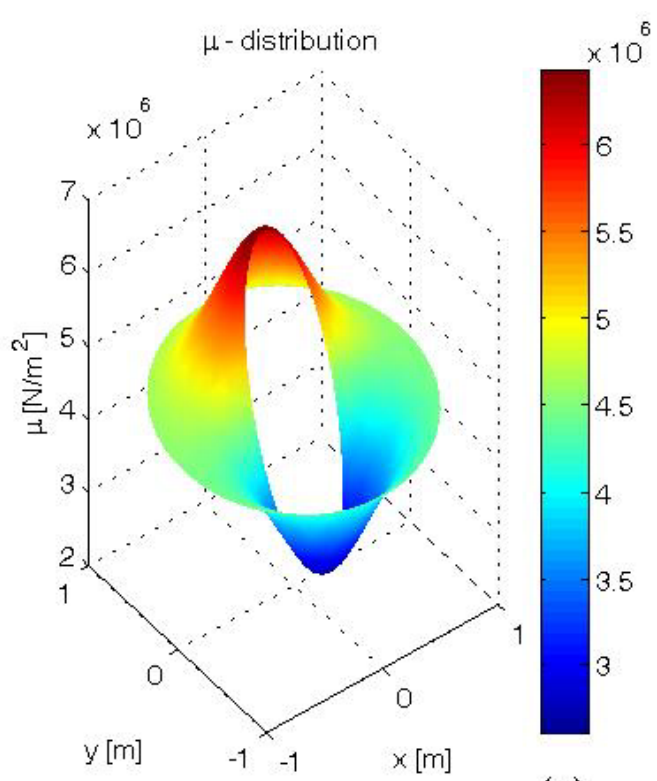

(a)

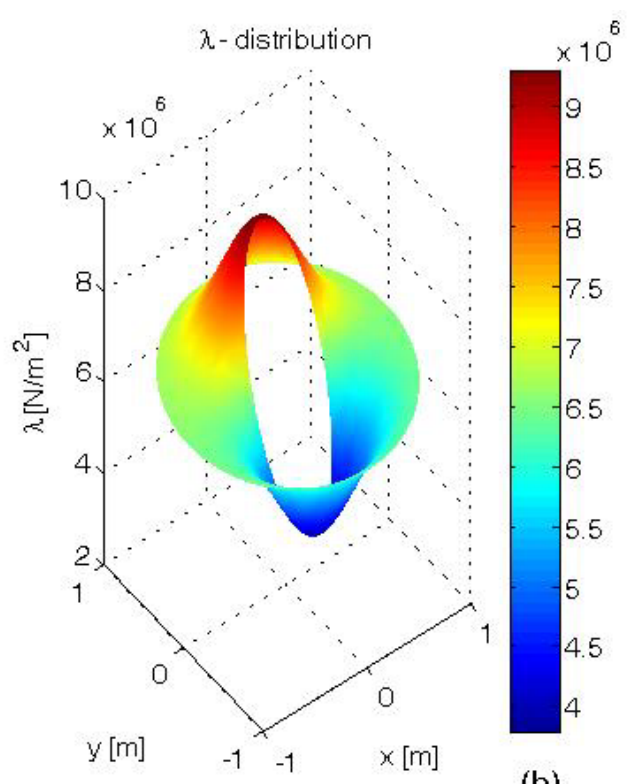

(b)

Figure 7. Spatial variation of Lame's constants for continuously nonhomogeneous material: a) $\mu$, b) $\lambda$

Homogeneous material properties of the cylinder with the constant value of Young's modulus $E^{i}(\mathbf{x})=E_{0}$ are considered first. As mentioned before, in the 2.5D analysis it is possible to expand the solution in the longitudinal direction using the spatial transform

$$
u_{i}\left(\mathbf{x}, x_{3}, \omega\right)=\tilde{u}_{i}\left(\mathbf{x}, k_{z}, \omega\right) e^{-i k_{z} x_{3}}
$$

In this way it is possible to specify the displacement response in any point in the longitudinal direction of the cylinder. A set of reference points is thus defined within $x_{3}=(0.0,5.9 \mathrm{~m})$. Figures 8 to 10 present the displacement responses in all three dimensions. Evolution of the response in the longitudinal direction can be clearly observed. 


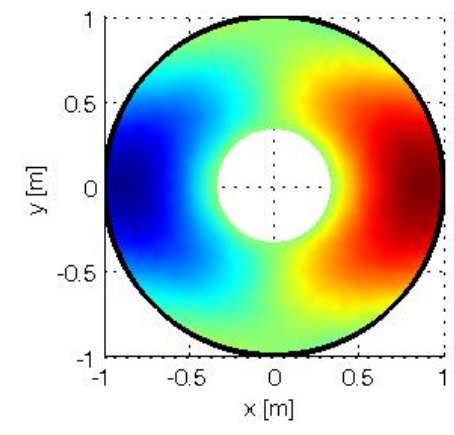

(a)

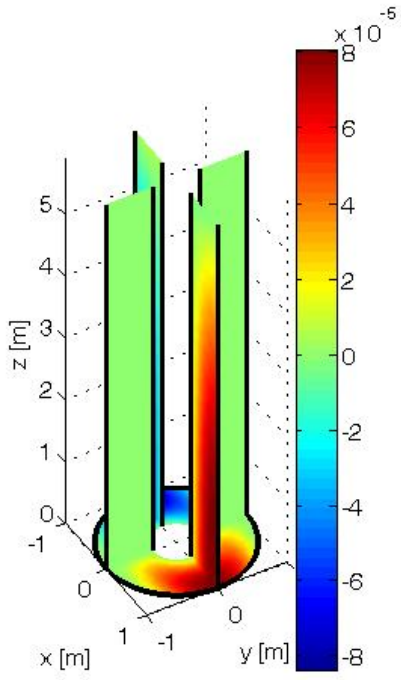

(b)

Figure 8. Numerical 2.5D response of displacement $\tilde{u}_{1}\left(\mathbf{x}, k_{z}, \omega\right)$ for homogeneous hollow cylinder: a) nodal solution in the cross-section of the cylinder b) expanded solution in longitudinal direction

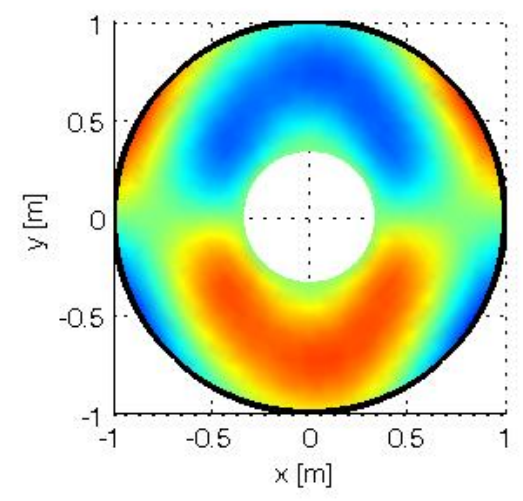

(a)

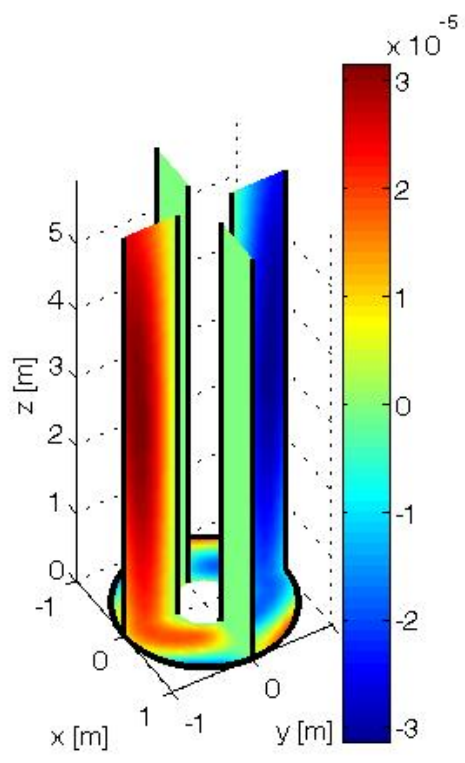

(b)

Figure 9. Numerical 2.5D response of displacement $\tilde{u}_{2}\left(\mathbf{x}, k_{z}, \omega\right)$ for homogeneous hollow cylinder: a) nodal solution in the cross-section of the cylinder b) expanded solution in longitudinal direction 


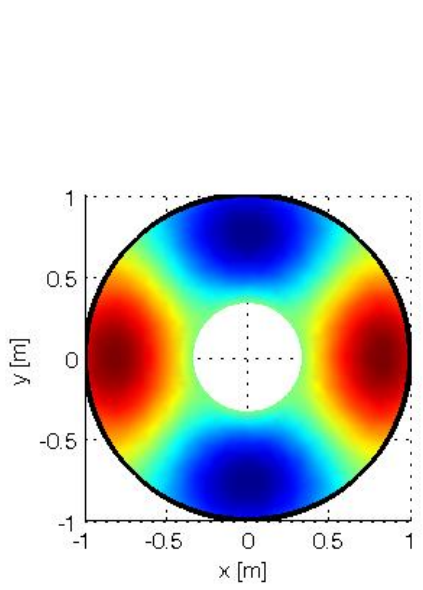

(a)

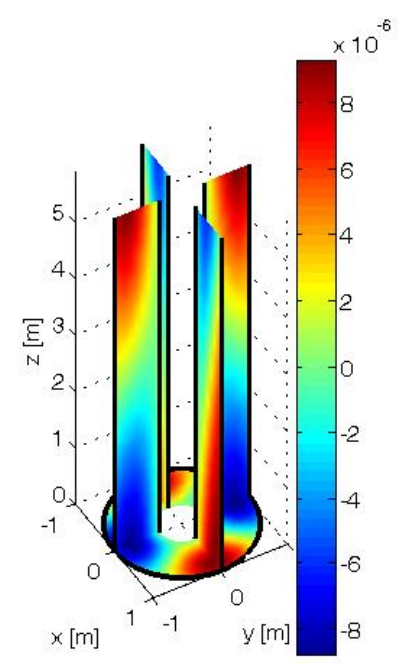

(b)

Figure 10. Numerical 2.5D response of displacement $\tilde{u}_{3}\left(\mathbf{x}, k_{z}, \omega\right)$ for homogeneous hollow cylinder: a) nodal solution in the cross-section of the cylinder b) expanded solution in longitudinal direction

Nonhomogeneous material properties are considered next with variation of Young's modulus given by Eq. (21). Figures 11 to 13 present the displacement responses in all three dimensions. Effect of varying material properties is clearly visible compared to Figures ( 8 - 10) for homogeneous material. Larger displacements are observed at the bottom part of the cylinder where the material properties are smaller.

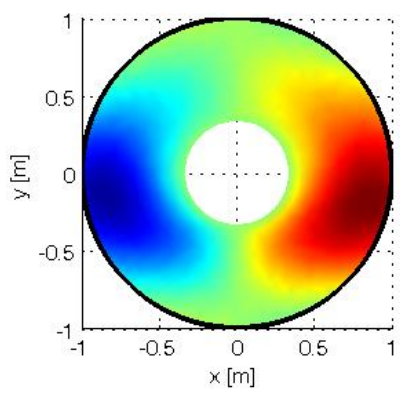

(a)

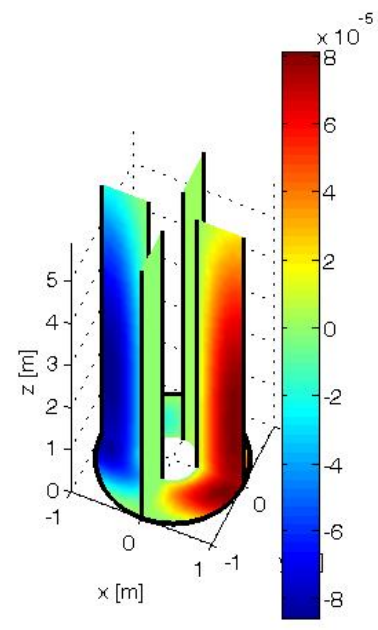

(b)

Figure 11. Numerical 2.5D response of displacement $\tilde{u}_{1}\left(\mathbf{x}, k_{z}, \omega\right)$ for nonhomogeneous hollow cylinder: a) nodal solution in the cross-section of the cylinder b) expanded solution in longitudinal direction 


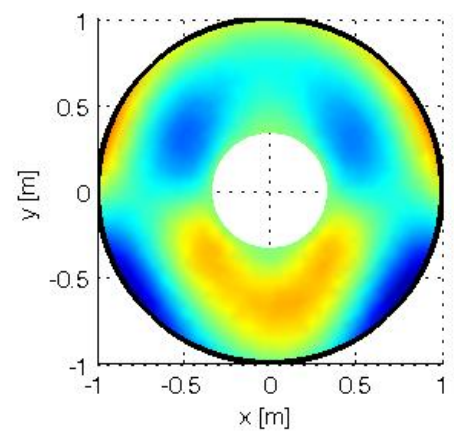

(a)

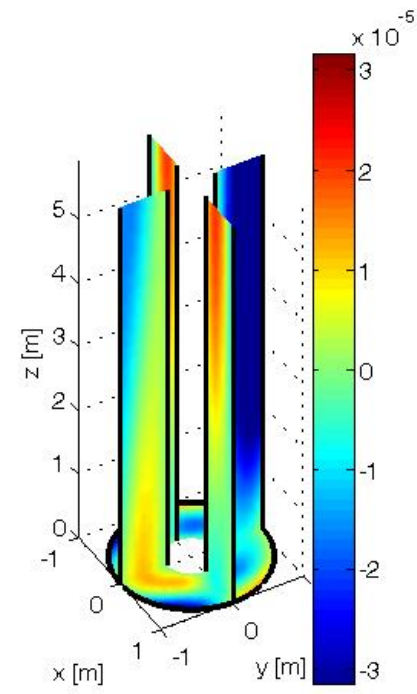

(b)

Figure 12. Numerical 2.5D response of displacement $\tilde{u}_{2}\left(\mathbf{x}, k_{z}, \omega\right)$ for nonhomogeneous hollow cylinder: a) nodal solution in the cross-section of the cylinder b) expanded solution in longitudinal direction

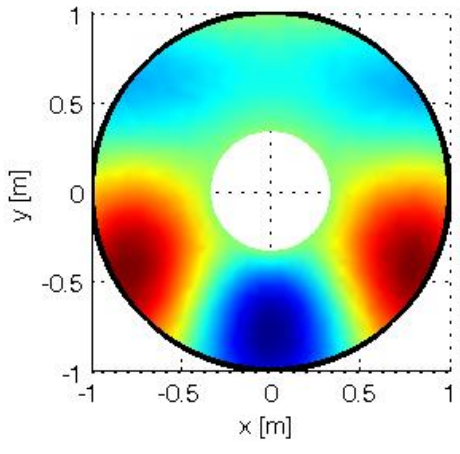

(a)

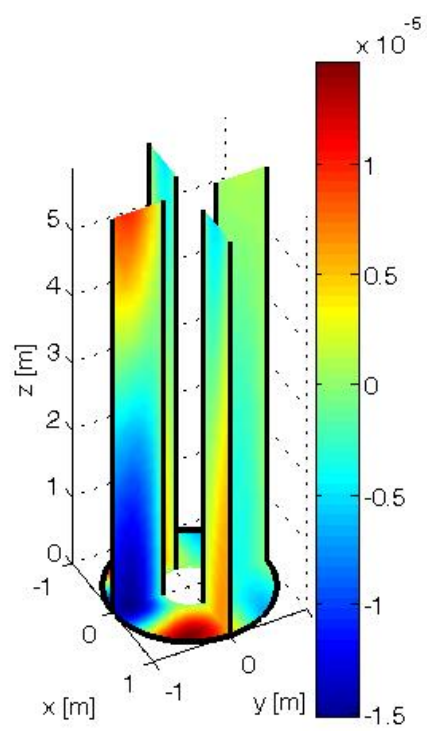

(b)

Figure 13. Numerical 2.5D response of displacement $\tilde{u}_{3}\left(\mathbf{x}, k_{z}, \omega\right)$ for nonhomogeneous hollow cylinder: a) nodal solution in the cross-section of the cylinder b) expanded solution in longitudinal direction 
The presented solution can be further extended for transient time analyses. Time responses can be obtained by applying an inverse Fourier transformation to computed set of spatial responses over a specified frequency range. This approach however requires significant computational power if the number of frequencies required is large.

\section{Conclusions}

A meshless local Petrov-Galerkin method (MLPG) is proposed for the solution of boundary value problems for elastic wave propagation in longitudinally invariant nonhomogeneous solids. The proposed method is a truly meshless method as no discretization elements were used for the approximation or integration of unknowns. The analyzed domain is divided into small overlapping spherical subdomains. The MLS approximation scheme has been used for approximation of trial functions. Using the Heaviside unit step function as a test function the boundary-domain formulation on each local subdomain has been obtained.

The patch test analysis has showed a high accuracy of the present method. Applicability of the proposed meshless method is demonstrated on numerical examples assuming continuously nonhomogeneous material properties and non-uniform mechanical displacement loading. Collocation of the applied displacement field using the MLS approximations is simple and straightforward.

A numerical application was used to illustrate the applicability of the proposed model for a hollow cylinder with variable elastic material properties. The analysis of displacement responses was found to be consistent with the physics of the problem.

\section{Acknowledgement}

The authors gratefully acknowledge the support by the Slovak Science and Technology Assistance Agency registered under number APVV-0014-10.

\section{References}

[1] Achenbach, J. D. (1973), Wave Propagation in Elastic Solids. North-Holland, Amsterdam.

[2] Aliabadi, M. H. (ed.). (2002), The boundary element method: applications in solids and structures. John Wiley and Sons, Chichester.

[3] Atluri, S. N., Sladek, J., Sladek, V. and Zhu, T. (2000), "The local boundary integral equation (lbie) and it's meshless implementation for linear elasticity“, Computational mechanics, 25, 180-198.

[4] Atluri, S. N. (2004), "The meshless method (MLPG) for domain \& BIE discretizations". Tech Science Press, Forsyth, USA.

[5] Bedford, A. and Drumheller, D. S. (1994), Introduction to Elastic Wave Propagation. Wiley, New York.

[6] Berezovski , A., Berezovski, M. and Engelbrecht, J. (2009), "Waves in Inhomogeneous Solids“. In: E. Quak, T. Soomere (eds.), Applied Wave Mathematics, 55-81.

[7] Carcione, J. M., Kosloff, D. and Kosloff, R. (1988), "Wave propagation simulation in an elastic anisotropic (transversaly isotropic) solid“. Quarterly Jnl. of Mechanics \& App. Math., 41(3), 319-346. 
[8] Dong, L., Alotaibi, A., Mohiuddine, S. A. and Atluri, S. N. (2014), “Computational Methods in Engineering: A Variety of Primal \& Mixed Methods, with Global \& Local Interpolations, forWell-Posed or Ill-Posed BCs“. CMES:Computer Modeling in Engineering \& Sciences, 99(1), 1-84.

[9] François, S., Schevenels, M., Galvín, P., Lombaert, G. and Degrande G. (2010), “A 2.5D coupled FE-BE methodology for the dynamic interaction between longitudinally invariant structures and a layered halfspace“. Comput. Methods Appl. Mech. Engrg., 199, 15361548.

[10] Hung, H. H., Chen, G. H. and Yang, Y. B. (2013), "Effect of railway roughness on soil vibrations due to moving trains by 2.5D finite/infinite elements“. Engineering Structures, 57, 254-266.

[11] Lancaster, P. and Salkauskas, T. (1981), "Surfaces generated by moving least square methods”, Math. Comput. 37, 141-158.

[12] Sheng, X., Jones, C. J. C. and Thompson, D. J. (2006), "Prediction of ground vibrations from trains using the wavenumber finite and boundary element methods“. Journal of Sound and Vibration, 293, 575-586.

[13] Sladek, J., Sladek, V. and Zhang, Ch. (2003), “Application of meshless local PetrovGalerkin (MLPG) method to elastodynamic problems in continuously nonhomogeneous solids“, CMES-Computer Modeling in Engineering and Sciences, 4, 637-648.

[14] Sladek, J., Stanak, P., Han, Z. D., Sladek, V. and Atluri S. N. (2013b), “Applications of the MLPG Method in Engineering \& Sciences: A Review”, CMES: Computer Modeling in Engineering \& Sciences, 92(5), 423-475.

[15] Sladek, J., Sladek, V., Solek, P. and Saez, A. (2008), "Dynamic 3D axisymmetric problems in continuously non-homogeneous piezoelectric solids”, International Journal of Solids and Structures 45, 4523-4542.

[16] Sladek, J., Sladek, V., Stanak, P., Zhang, Ch. and Wünsche, M. (2013a), “Analysis of the bending of circular piezoelectric plates with functionally graded material properties by a MLPG method“, Engineering Structures, 47, 81-89.

[17] Sladek, J., Sladek, V., Wunsche, M. and Zhang, C. (2009), "Interface crack problems in anisotropic solids analyzed by the MLPG“. CMES-Computer Modeling in Engineering \& Science, 54, 223-252.

[18] Sladek, V., Sladek, J. and Tanaka, M. (2005), "Local Integral Equations and two Meshless Polynomial Interpolations with Application to Potential Problems in Nonhomogeneous Media“, CMES- Computer Modeling in Engineering \& Sciences, 7, 69-83.

[19] Stanak, P., Sladek, J., Sladek, V. and Krahulec, S. (2011), “Composite circular plate analyzed as a 3-D axisymmetric piezoelectric solid“, Building Research Journal, 59(3-4), 125-140.

[20] Stanak, P., Sladek, V., Sladek, J., Krahulec, S. and Sator, L. (2013), “Application of patch test in meshless analysis of continuously non-homogeneous piezoelectric circular plate“, Applied and Computational Mechanics, 7(1), 65-76.

[21] Tadeu, A. and Antonio, J. (2001), “2.5D Green’s functions for elastodynamic problems in layered acoustic and elastic formations“, CMES- Computer Modeling in Engineering and Sciences, 2, 477-495.

[22] Tadeu, A., Kausel, E. and Vrettos, C. (1996), "Scattering of waves by subterranean structures via the boundary element method“, Soil Dyn Earthquake Eng., 15(6), 387-397. 
[23] Tadeu, A. and Kausel, E. (2000), “Green’s functions for two-and-a-half-dimensional elastodynamic problems“, ASCE J. Engrg. Mech., 126(10), 1093-1096.

[24] Tadeu, A., Stanak, P., Sladek, J. and Sladek, V. (2014), “Coupled BEM-MLPG acoustic analysis for non-homogeneous media“, Engineering Analysis with Boundary Elements, 44, 161-169.

[25] Zhang, C. and Zhao, C. (1987), “Coupling method of finite and infinite elements for strip foundation wave problems“, Earthquake Engineering and Structural Dynamics, 15, 839851. 